\title{
Enabling a Wind Energy Harvester Based on ZnO thin Film as the
}

\author{
Building skin \\ LiDong Du ${ }^{\text {a, }}{ }^{*}$, Fang Zhen ${ }^{\text {a, c }}$, Jize Yan ${ }^{\mathrm{b}}$, Zhan Zhao ${ }^{\mathrm{a}, \mathrm{c}}$ \\ ${ }^{\text {a }}$ State Key Laboratory of Transducer Technology, Institute of Electronics, Chinese Academy of Sciences, Beijing \\ 100190, China \\ ${ }^{\mathrm{b}}$ Nano Group, Electronics and Computer Science, University of Southampton, Southampton SO17 1BJ, UK \\ ${ }^{c}$ University of Chinese Academy of Sciences, Beijing 100049, China
}

\begin{abstract}
In this paper, a wind energy harvester, which can be used as building skin, is developed. In order to overcome the defects of traditional wind energy harvester, such as expensive and crisp, the developed harvester is designed as a circular silicon plate with several symmetrically distributed rectangular cavities which were covered with $\mathrm{ZnO}$ (Zinc oxide) thin film. It is verified whether the designed harvester can function in turbulence flow by theoretical analysis and finite element analysis. The acoustic measurement illustrated that the output of designed device is linear to the frequency. And, the wind fluid experiments suggested that the thin film can vibrate under a low wind velocity $1 \mathrm{~m} / \mathrm{s}$ and is easy to vibrate in wind turbulence. The maximum output power density is $23.39 \mathrm{nW} / \mathrm{cm}^{2}$ with a maximum open circuit output voltage $2.81 \mathrm{~V}$. All of characteristics of designed harvester enable that it can be used as the building skin to harvest wind energy.
\end{abstract}

Keywords Wind energy harvester, $\mathrm{ZnO}$ thin film array, fluid-structure interaction

\section{Introduction}

Nowadays, wind energy has been extensively utilized in suburban in the world. Many windmills have greatly facilitated people's life. However, wind energy nearly has not been used yet in urban. The existed wind energy harvester cannot be directly used to cover the outside-surface of building, such as windmill or turbine [1-5], due to huge volume, expensive cost and complex design. Recently, several kinds of miniaturized wind energy harvesters have been developed such as the cm-scale shrouded wind turbine [6], the wake galloping energy harvesting system [7], arc-shaped piezoelectric generator [8], piezoelectric reed and resonant cavity device, piezoelectric windmill and piezo-leaf [9-12]. But they are too crisp or too complicated to use. As the skin of building the wind energy harvester should be cost-effective for large-area covering. If a kind of wind energy harvester, which can be used on the outside-surface of building, can be developed it will greatly promote the utilization of wind energy in city.

MEMS (Microelectromechanical systems) technology merely meets the requirement of developing cost-effective wind energy harvester due to the advantages of batch fabrication, good consistency and more convenience. In the MEMS zone the wind energy should be transformed to vibration energy before it can be used. Therefore, the design of vibrated structure becomes the critical part of developing a wind energy harvester. Normally, cantilever/beam [13-16], coil and film are common used in a vibration energy harvester. But cantilever/beam is too crisp to apply in outside-surface of building. And, the coil principle vibration harvester is complex to convert wind energy into vibration energy. Thus, film structure becomes the best choice for energy harvester as building skin. But a robust structure is still critical.

The piezoelectric materials of energy harvester are important in developing a wind energy harvester as well. Traditionally, zinc oxide ( $\mathrm{ZnO}$ ) [17], lead zirconate titanate (PZT) [18], lead magnesium niobate-lead titanate

\footnotetext{
* Corresponding author: lddu@mail.ie.ac.cn
} 
(PMN-PT) [19], barium titanate $\left(\mathrm{BaTiO}_{3}\right)$ [20], zinc stannate $\left(\mathrm{ZnSnO}_{3}\right)$ [21], alkaline niobate-based particles (KNLN) with copper $(\mathrm{Cu})$ nanorod filler [22], have been used as inorganic piezoelectric material due to high piezoelectric coefficient. Meanwhile, organic piezoelectric materials are used as well because of the enhanced efficient of energy harvester such as polyvinylidenefluoride (PVDF) based polymer materials [23-27], $\mathrm{Na}_{0.47} \mathrm{~K}_{0.47} \mathrm{Li}_{0.06} \mathrm{NbO}_{3}$ (NKLN) based polydimethylsiloxane (PDMS) polymer [28] and biomaterial based polydimethylsiloxane (PDMS) polymer [29]. However, $\mathrm{ZnO}$ is one of the most common used among these material due to abundant in nature, low cost and easy to fabricate. Thus, $\mathrm{ZnO}$ becomes the best material for energy harvester as building skin.

In our previous work [30], MEMS based device with $\mathrm{ZnO}$ thin film has been developed to meet the requirement of wind energy harvesting on outside surface of building. The developed harvester is designed as a circular silicon plate with several symmetrically distributed rectangular cavities which were covered with $\mathrm{ZnO}$ thin film. When the flow blows to the harvester, it can produce energy. In this paper, based on the previous results the principle is totally established and the results are specifically analyzed. It is verified whether the designed harvester can function in turbulence flow by theoretical analysis, finite element analysis. Meanwhile, the results are verified and explained by simulation and the Experiments in wind tunnel and acoustic tube.

\section{Principle and Fabrication}

\subsection{Principle design}

According to R.D. Blevins [31], structure will perform at its resonant state when it is immersed into turbulence. The resonant frequency will be modulated by the interaction between structure and the turbulence. Thus it is feasible to develop a wind energy harvester based on this phenomenon. With the increasing of flow speed, the shear stress on the structure will increase. It will eventually increase the resonant frequency. Basically, it is reasonable that the harvester efficiently functions at the fundamental resonant frequency. But, the efficiency of energy harvesting will decrease when the resonant frequency exceeds second mode. That is, harvester should avoid to work on the second-mode resonant frequency. On the other hand, harvester should be strong enough to resist the disturbance of ambient dust and have high sensitivity and energy capacity. However, robustness, sensitivity and energy capacity are mutually restrained. Larger and thinner film, which has high energy capacity and large flexibility, can enable high sensitivity to the wind. But, the larger and thinner film device has poor robustness. Fortunately, thin film array is suitable to meet the requirements of robustness, sensitivity and energy capacity. Therefore, in this paper we design a harvester based on a circular silicon plate structure on which several rectangular cavities are symmetrically distributed. The thin film array is formed by these cavities which are covered with $\mathrm{ZnO}$ thin film. In this structure, the total effective energy-harvesting area is increased; single film could be small enough to ensure stability; and the silicon frame between films could also enhance the robustness of the device. That means the mutual restraint can be resolved effectively. The principle of harvester, which is based on the designed structure, is shown in Fig. 1.

The harvester is mainly effected by the flow-drag force, which will cause the change of shear stress, when it is immersed in wind flow. Furthermore, the shear stress will affect the resonant frequency. Thus it is significant to figure out the relationship between the flow-drag force and resonant frequency.

Pressure distribution, which depends on flow, can be expressed by equation (1) [32].

$$
\mathrm{P}_{\text {fluid }}=\frac{1}{2} \rho_{F} V^{2}
$$

Where $\rho_{\mathrm{F}}$ and $\mathrm{V}$ are fluid density and flow velocity. When Re, which is the Reynolds Number, is higher than 1000 , the $C_{D}$, which is the coefficient of drag force, is between 1.28 2.0. When the flow-drag force is exerted on a harvester, there will be a pressure distribution around the harvester which will lead to the bend of thin film in harvester. Thus shear stress occurs on the thin film. Shear stress can be calculated by the pressure distribution [33].

$$
\sigma_{\max }=0.31 P \frac{a^{2}}{h^{2}}
$$




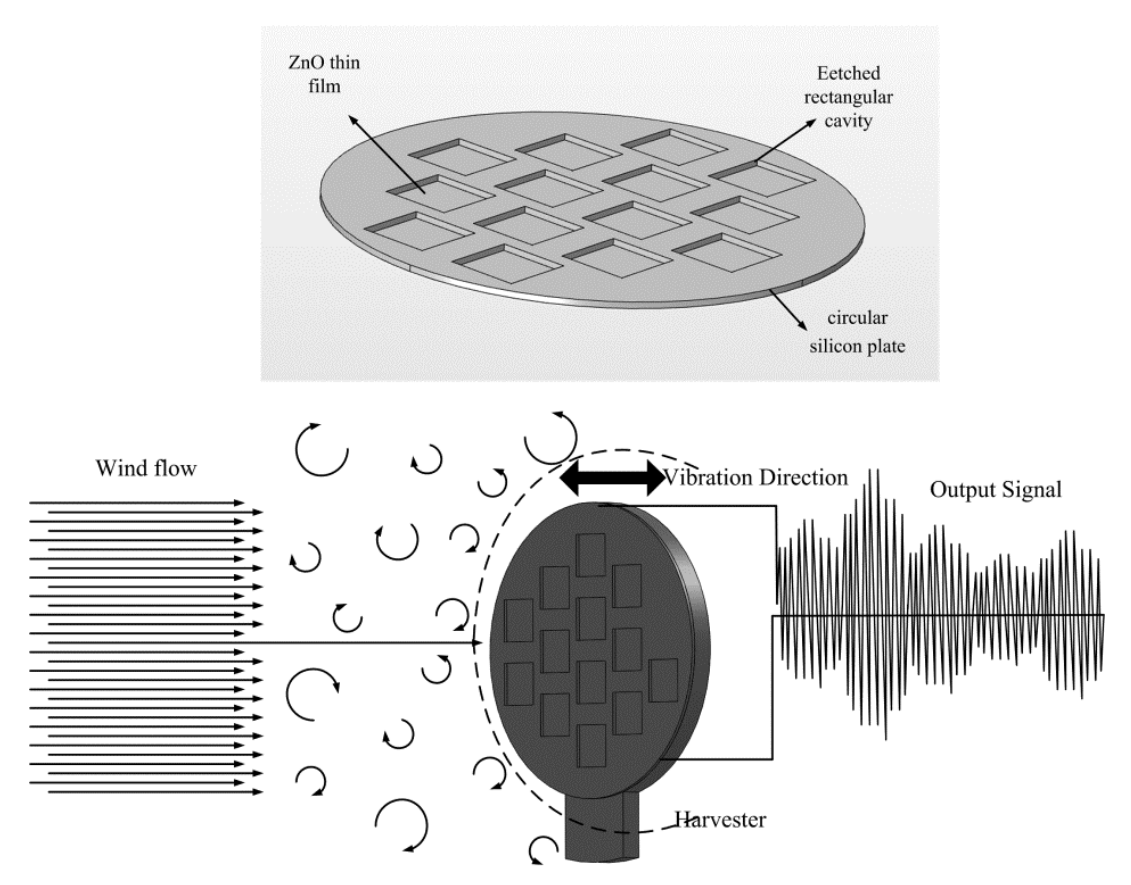

Fig. 1 The principle of harvester

Where $\mathrm{P}, \mathrm{A}_{\text {film }}$, a and $\mathrm{h}$ are the pressure, thin film area, length of rectangle cavity and thickness of thin film. Substituting equation (1) into equation (2), the relationship between shear stress and flow-drag force can be deduced by equation (3).

$$
\sigma_{\max }=0.155 \rho_{F} V^{2} \frac{a^{2}}{h^{2}}
$$

The dependence of resonance frequencies on stress has been extensively studied and simulated [34]. Several methods have been proposed to solve the problem of determining the natural frequencies and mode shapes of thin film $[35,36]$. Resonant frequency of square thin film can be expressed by [36]

$$
f_{n, m}=\frac{1}{2} \sqrt{\frac{\delta_{\max }}{\rho}} \sqrt{\frac{n^{2}+m^{2}}{a^{2}}}
$$

When $n=m=1, f_{11}$ is the fundamental resonant frequency. Substituting equation (3) into equation (4), the resonant frequencies with residential shear stress can be expressed by equation (5).

$$
f_{n, m}=\frac{1}{2} \sqrt{\frac{\delta_{\max }}{\rho_{s}}} \sqrt{\frac{n^{2}+m^{2}}{a^{2}}}=\frac{1}{2} \sqrt{\frac{0.155 V^{2}\left(n^{2}+m^{2}\right)}{h^{2}} \frac{\rho_{F}}{\rho_{s}}}
$$

When the wind velocity and thickness of the thin film are specific the resonant frequency will be explicit. In the designed device structure, the thickness of $\mathrm{ZnO}$ thin film is much less than that of circular silicon plate. Therefore, it is believed that the energy is harvested due to the vibration of $\mathrm{ZnO}$ thin film. In a word, from the theoretical point of view the designed harvester can function well.

\subsection{Fabrication}

The fabrication schematics and thin film array are illustrated in Fig. 2. The fabrication processes are based on a standard 4 inch MEMS streamline. The thin film of device includes $\mathrm{SiO}_{2}$ (Silicon dioxide) layer, $\mathrm{Si}_{3} \mathrm{~N}_{4}$ ( $(\mathrm{Silicon}$ nitride) layer, bottom platinum layer, $\mathrm{ZnO}$ layer, and up platinum layer in turn from the bottom to top. $\mathrm{SiO}_{2}$ and $\mathrm{Si}_{3} \mathrm{~N}_{4}$ layers are grown on the surfaces of silicon wafer at the beginning of the fabrication which thickness are $300 \mathrm{~nm}$ and $200 \mathrm{~nm}$ respectively. Both of them will play a role of insulator and match thermal residential stress. Then lithography and wet etching processes are conducted to produce square cavity arrays from the backside of the wafer. Afterward a bottom platinum layer with $300 \mathrm{~nm}$ thickness is sputtered as the electrode and pads on front side 
of the wafer. Subsequently $\mathrm{ZnO}$ layer with thickness $300 \mathrm{~nm}$ is sputtered with MSPVD (Magnetron Sputtering Physical Vapor Deposition) process. After that, up platinum layer is sputtered onto the ZnO layer. At last, the DRIE (deep iron reactive etching) process is adopted to release the films by removing the residual silicon in the square cavities from the backside.

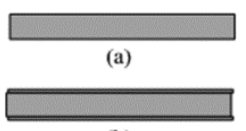

(b)

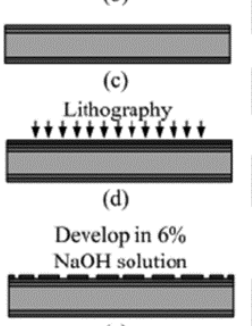

(e)

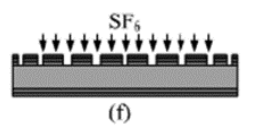

$\square \mathrm{Si} \square \mathrm{SiO}_{2} \square \mathrm{Si}_{3} \mathrm{~N}_{4} \square$
Wet etching in 33\%

KOH solution

周目用同同

$(\mathrm{g})$

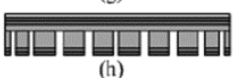

(h)

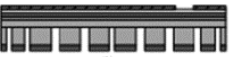

(i)

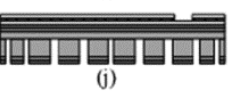

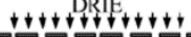

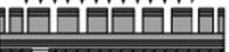

(k)

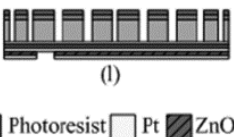

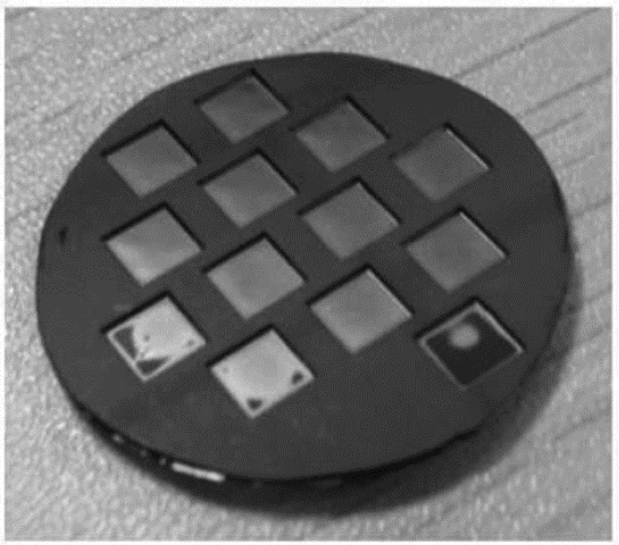

Fig. 2 The fabrication schematics and thin films array

It is important to select the highest piezoelectric constant of $\mathrm{ZnO}$ layer in designed harvester. For $\mathrm{ZnO}$ material the c-axis is chose for best piezoelectric properties due to the highest piezoelectric constant among all the crystal orientation. As shown in Fig. 3, the $\mathrm{ZnO}$ film obtained highly c-axis-oriented polycrystalline structure.

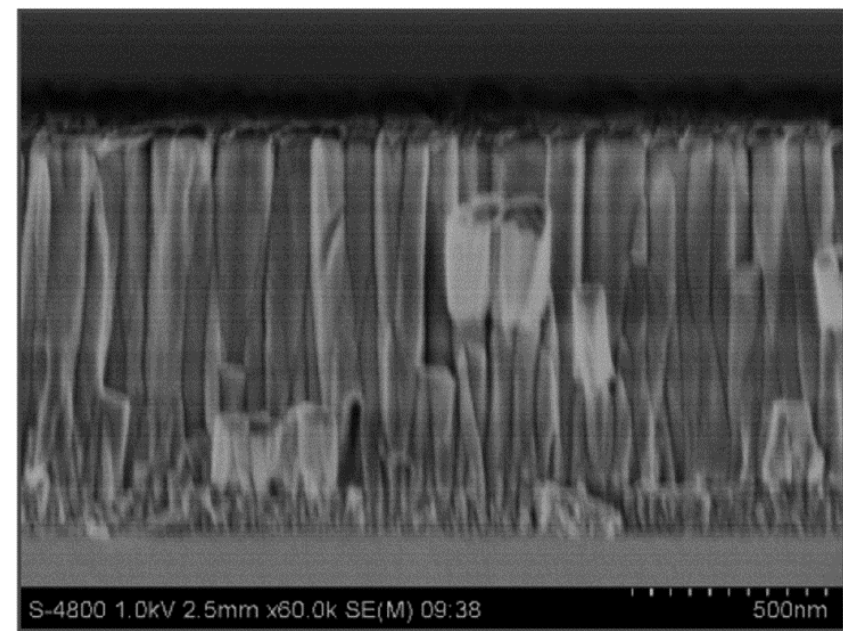

Fig. 3 Scanning electron microscope (SEM) photo of the $\mathrm{ZnO}$ film

The Profiler is used to measure the precise thickness of thin film. It is shown that the thickness is about $2 \mathrm{um}$ due to error of LPCVD (Low pressure chemical vapor deposition), sputter and DIRE process.

\subsection{Experimental design}

First of all, in order to validate the performance of the device with the variation of frequency, the developed harvester will be tested in an acoustic-electric measurement system AWA6122A which is supplied by Hangzhou AiHua Instruments Company with an acoustic noise source AWA77 speaker which frequency ranges from $1500 \mathrm{~Hz}$ 
to $6500 \mathrm{~Hz}$.

Secondly, the wind fluid experiments will be carried out in self-manufactured small wind tunnel which is shown in Fig. 4. In the setup, the wind velocity is measured by anemometer KANOMAX 6004, and the wind energy harvesting device is placed above the anemometer.
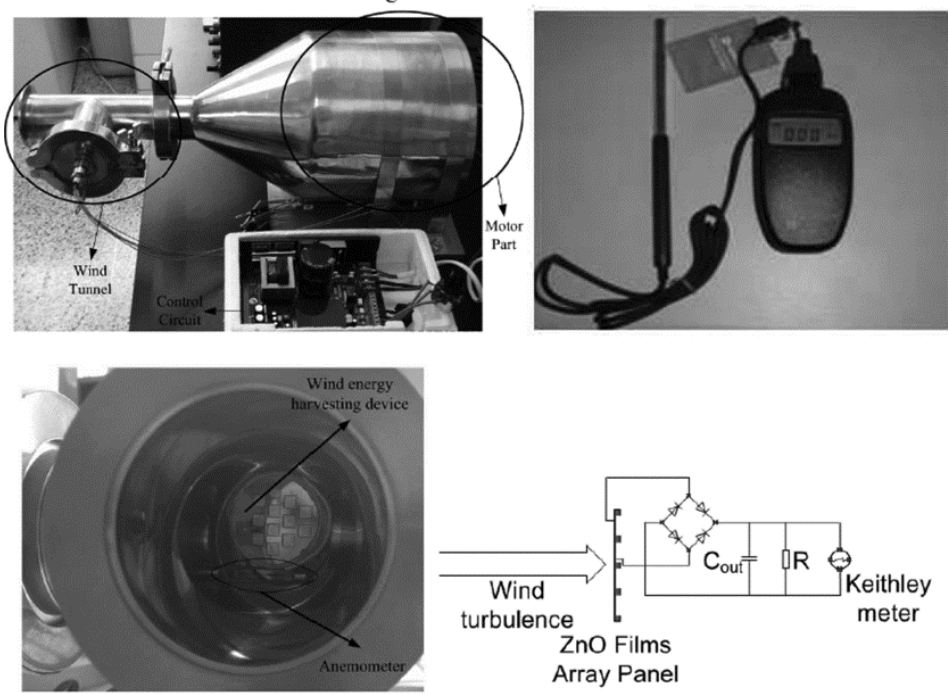

Fig. 4 Wind flow test equipment and energy convert circuit

It is important how the small wind tunnel can work in a turbulent state. Reynolds Number is an important factor for judgement which can be calculated with the following equation (6).

$$
R e=U \frac{d}{\vartheta}
$$

The parameter $v$ is the air viscous friction coefficient. The diameter $\mathrm{d}$ of wind tunnel is equal to $45.2 \mathrm{~mm}$. The Reynolds Number of small wind tunnel is more than 2300 when the mean speed of the wind flow is more than $0.799 \mathrm{~m} / \mathrm{s}$. It is suggested that the equipment mainly works in turbulence state.

\section{Simulation}

In order to prove the effectiveness of harvester, it is also important to run simulations in finite element analysis field. Some important parameters, which are listed in table 1, should be fixed during the FEA (Finite element analysis) simulations.

Table 1. The parameters using in calculation and simulation

\begin{tabular}{|c|c|c|c|c|c|c|}
\hline item & Silicon & $\mathbf{Z n O}$ & Platinum & $\mathbf{S i O}_{\mathbf{2}}$ & $\mathbf{S i}_{\mathbf{3}} \mathbf{N}_{\mathbf{4}}$ & air \\
\hline Density $\left(\mathbf{K g} / \mathbf{m}^{\mathbf{3}}\right)$ & 2331 & 5676 & 21450 & 2200 & 3100 & 1.29 \\
\hline Young's modulus & $130 \mathrm{E} 9$ & $210 \mathrm{E} 9$ & $168 \mathrm{E} 9$ & $70 \mathrm{E} 9$ & $250 \mathrm{E} 9$ & \\
\hline Poisson's ratio & 0.27 & 0.33 & 0.39 & 0.17 & 0.23 & \\
\hline Thickness of square thin film & & $0.3 \mu \mathrm{m}$ & $0.3 \mu \mathrm{m}$ & $0.3 \mu \mathrm{m}$ & $0.2 \mu \mathrm{m}$ & \\
\hline Length of square ZnO thin film & \multicolumn{7}{|c|}{$4 \mathrm{~mm}$} \\
\hline Thickness of circular silicon plate & \multicolumn{7}{|c|}{$14 \mathrm{~mm}$} \\
\hline Diameter of circular silicon plate & \multicolumn{7}{|c|}{} \\
\hline
\end{tabular}

The first step of FEA simulation is to obtain the resonant frequency in different mode without residential stress. The structure module in COMSOL software is used to run eigenfrequency simulation which is shown in Fig. 5. It is shown the fundamental resonant frequency is $725.44 \mathrm{~Hz}$ and the second mode resonant frequency 3640.9 Hz. Such frequency range enables the thin film easy to vibrate. It is also suggested that the harvested energy will 
drastically plummet when the resonant frequency is more than the second resonant frequency.

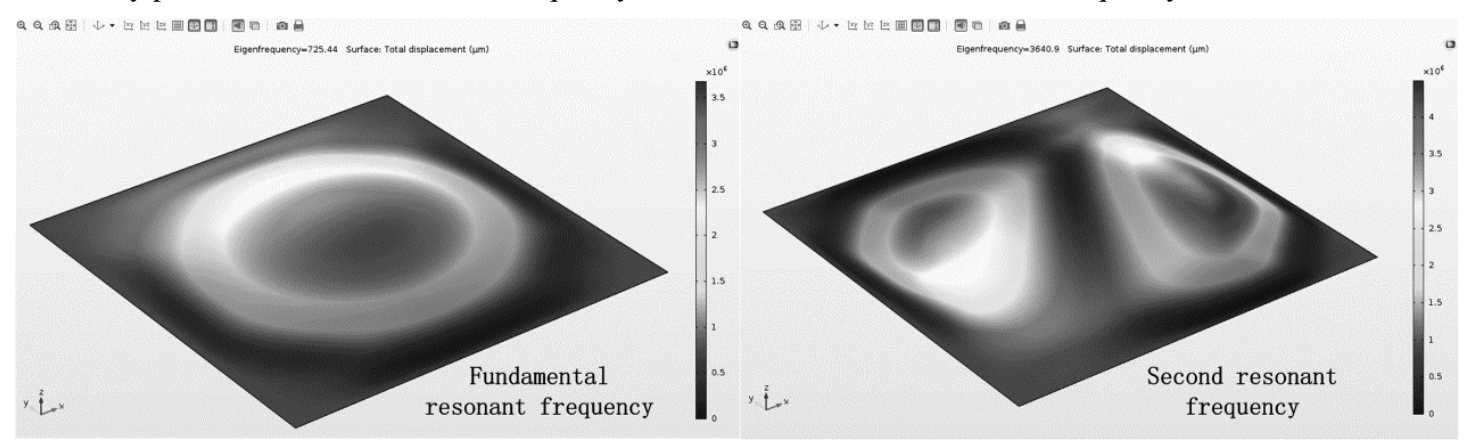

Fig. 5 Eigenfrequency simulation in COMSOL

According to the theoretical analysis resonant frequency will be affected by stress. And the incident flow will lead to the deflection of thin film and induce stress on the thin film. Therefore, it is indispensable to simulate the variation of resonant frequency under variation of flow velocity. But it is impossible to directly simulate the resonant frequency with change of flow velocity in COMSOL. However, it is feasible to simulate the variation of stress under the variation of flow velocity by the flow-structure interaction module in COMSOL. When the stress results are obtained the resonant frequency can be calculated by equation (4). During the simulation the effective Young's modulus, Poisson's ratio and density are used in order to simplify the simulation of the multilayer of the thin film.

The effective density can be deduced by equation (7).

$$
\rho_{\text {eff }}=\frac{\sum_{k} \rho_{k h_{k}}}{h_{\text {total }}}
$$

The effective Young's modulus can be approximately deduced by equation (8) [37]. Where $V_{k}$ is the volume of each layer $\mathrm{V}_{\text {total }}$ is the total volume of thin film.

$$
E_{\text {eff }}=\sum_{k} E_{k} \frac{V_{k}}{V_{\text {total }}}
$$

The value of effective Poisson's ratio is set to 0.23 which is between the minimal and maximal values of the Poisson's ratio of the individual components [38]. The effective young's modulus and density is set to 167.7E9Pa and $11388 \mathrm{Kg} / \mathrm{m}^{3}$, respectively. These three values are also validated by eigenfrequency simulation in COMSOL software. It is suggested by the simulation results that the fundamental resonant frequency of single layer, which uses the three effective value, is 794.28 which is close to the fundamental resonant frequency of five-layer thin film 725.44 .

The fluid-structure interaction simulations are conducted with those three effective parameters. During the simulation the transient flow is turn on and $\mathrm{k}-\varepsilon$ model is used in simulation model to mock the turbulence flow environment. At first, a 2D stationary and time-depend simulation are conducted which are shown in Fig. 6. In the model the flow channel shape is the cross-section view of test equipment which is shown in Fig. 4. The device was set at the location according to the test experiment. The fluid is air with standard density and dynamic viscosity in COMSOL. The rectangle structure in the middle of flow channel is the device with the effective Poisson's ratio, density and Young's modulus which were calculated before. The top and bottom edges of the rectangle are fixed to the flow channel that is oriented perpendicular to the fluid flow streamline. The outflow surface of the flow channel is set to a zero pressure. The velocity of flow channel is a mean value in stationary simulation and a step input in time-depend simulation. Compared the results of stationary and time-depend simulation, the designed device can definitely function in turbulence situation. Then the time-depend simulations were conducted with the wind velocity $1 \mathrm{~m} / \mathrm{s}, 4 \mathrm{~m} / \mathrm{s}, 6 \mathrm{~m} / \mathrm{s}, 8 \mathrm{~m} / \mathrm{s}$ and $10 \mathrm{~m} / \mathrm{s}$ which are shown in Fig. 7. The pressure distribution shows that 
the turbulence will enhance with the increase of wind velocity. That is the device can work in turbulence within the velocity range.

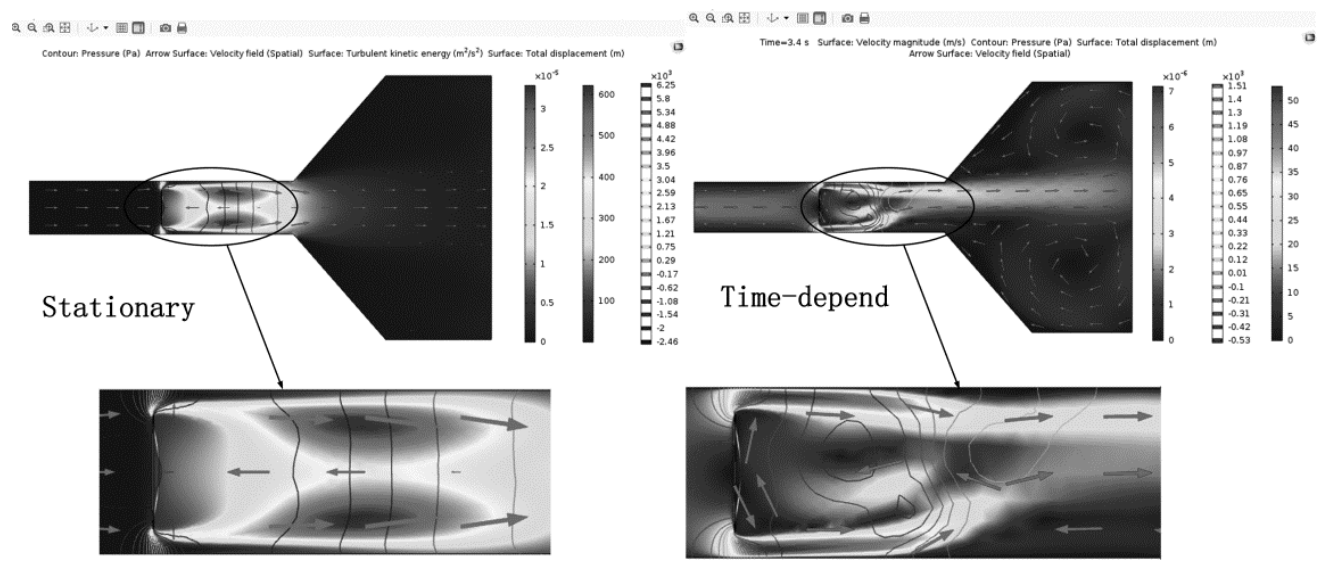

Fig. 6 2D stationary and time-depend simulation in COMSOL

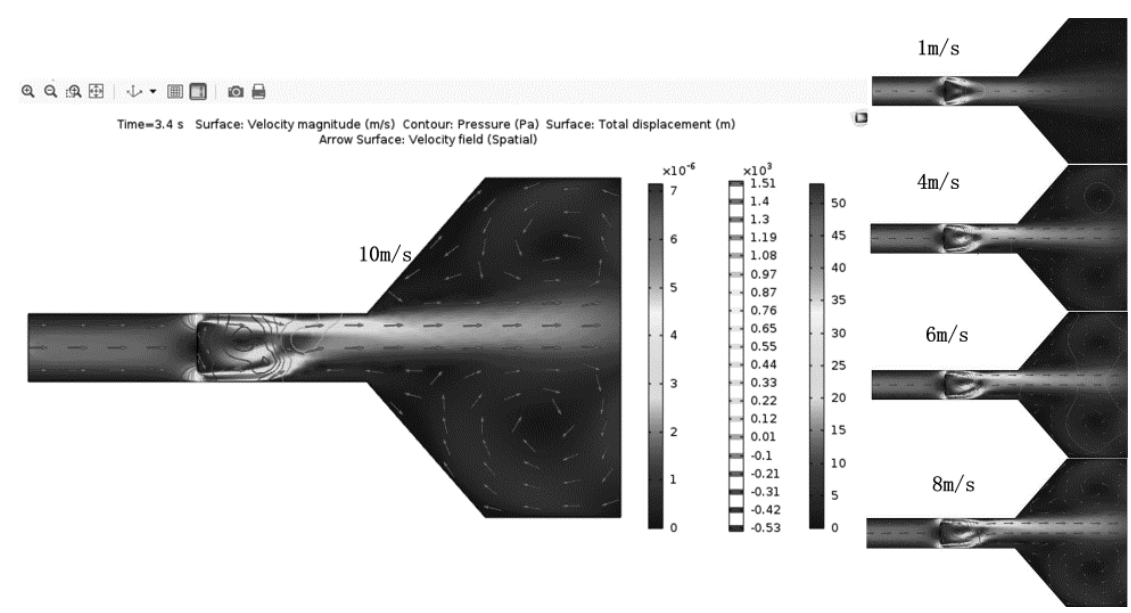

Fig. 7 Time-depend simulation with different wind velocity in COMSOL

Then the flow-structure interaction simulations were conducted which is shown in Fig. 8. The cylinder represents the flow channel. The fluid is air with standard density and dynamic viscosity in COMSOL. The plate structure is the solid part with the effective Poisson's ratio, density and Young's modulus which were calculated before. The edges of the plate are fixed to the flow channel that is oriented perpendicular to the fluid flow streamline. All other boundaries of the plate experience the load from the fluid. At the mean time, the outflow surface of the flow channel is set to a zero pressure. The velocity of flow channel is a mean wind velocity. And the turbulence conditions are exerted with intensity 0.05 and turbulence length scale $0.01 \mathrm{~m}$. The rest four surfaces of the fluid channel are defined as "no slip" condition, which means these surfaces serve like walls that confine the fluid flow between them. By conducting a series simulation under wind velocity range from $0.1 \mathrm{~m} / \mathrm{s}$ to $15 \mathrm{~m} / \mathrm{s}$, the maximum stresses on the thin film can be obtained and are listed in table 2. Then the maximum stresses were transferred to resonant frequency by equation (4).

At the same time, the theoretical resonant frequency is calculated which are listed in table 2 as well. It is shown that the theoretical results are less than the simulation results. It is mainly because that the FSI simulation is more depended on the dimensions of wind flow channel. The maximum stress will vary with the dimension. But it is also shown in table 2 that the resonant frequency will greatly change with the wind velocity variation. At this point of view, the simulation and theoretical calculation is consistent. 


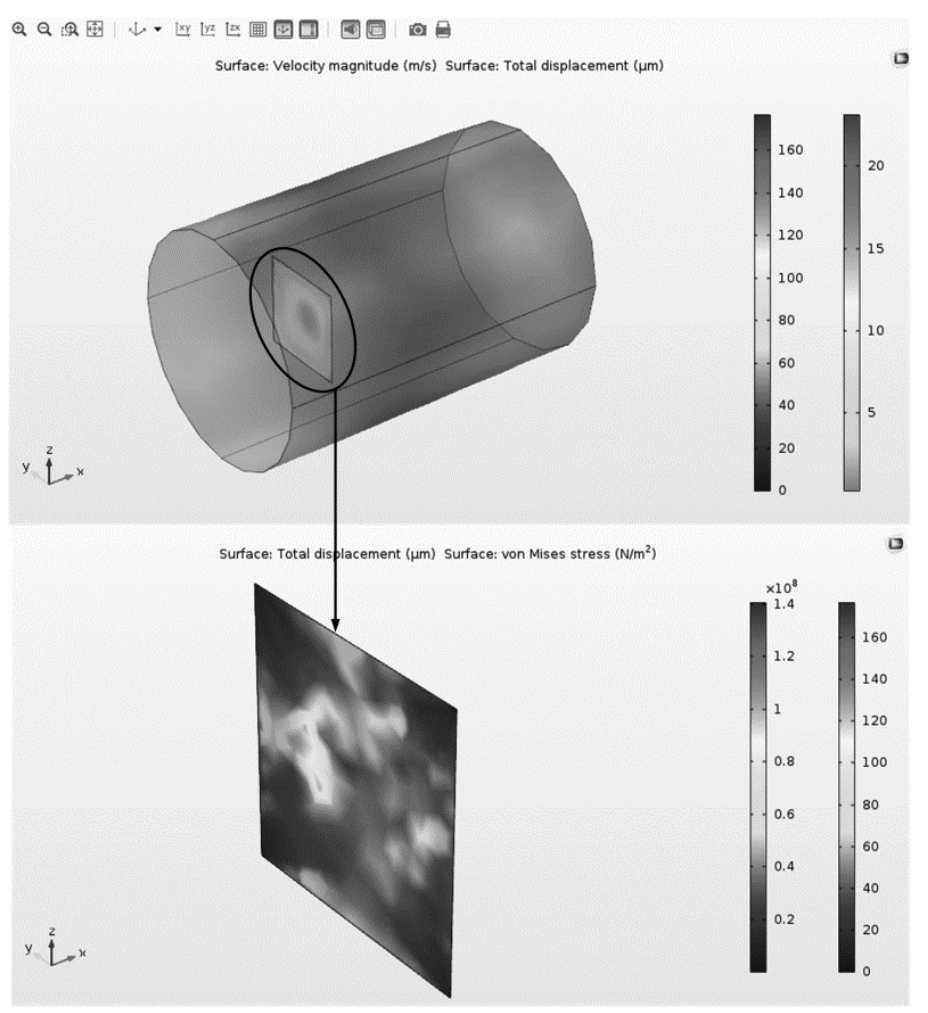

Fig. 8 Fluid-structure interaction simulation in COMSOL

Table 2 maximum stress on the thin film, deduce Resonant frequency and theoretically calculated Resonant frequency

\begin{tabular}{|c|c|c|c|}
\hline \multirow{2}{*}{ Velocity $(\mathbf{m} / \mathbf{s})$} & \multicolumn{2}{|c|}{ Simulated results } & Theoretical calculated results \\
\cline { 2 - 4 } & Maximum stress (Pa) & Resonant frequency $(\mathbf{H z})$ & Resonant frequency (Hz) \\
\hline 0.1 & $1 \mathrm{E} 4$ & 154 & 122 \\
\hline 1 & $5.5 \mathrm{E} 5$ & 1263 & 1218 \\
\hline 2 & $2.13 \mathrm{E} 6$ & 2484 & 2437 \\
\hline 3 & $4.84 \mathrm{E} 6$ & 3745 & 3655 \\
\hline 4 & $8.34 \mathrm{E} 6$ & 4918 & 4874 \\
\hline 5 & $1.38 \mathrm{E} 7$ & 6317 & 6092 \\
\hline 6 & $1.92 \mathrm{E} 7$ & 7459 & 7310 \\
\hline 7 & $2.54 \mathrm{E} 7$ & 8589 & 8529 \\
\hline 8 & $3.34 \mathrm{E} 7$ & 9836 & 9747 \\
\hline 9 & $4.17 \mathrm{E} 7$ & 10997 & 10966 \\
\hline 10 & $5.17 \mathrm{E} 7$ & 12246 & 12184 \\
\hline 11 & $6.17 \mathrm{E} 7$ & 13379 & 13402 \\
\hline 12 & $7.42 \mathrm{E} 7$ & 14672 & 14621 \\
\hline
\end{tabular}

\section{Results and Analysis}

First of all, the acoustic experiments were conducted by the acoustic-electric measurement system. The open circuit voltage of device ramps with the increasing of the frequency and the sounder voltage. The maximum voltage is $69.41 \mathrm{mV}$ at the largest frequency and sounder voltage given by the experiment which is shown in Fig. 9. It is clearly illustrated that the output of designed device is linear to the frequency and also linear to the magnitude of incident source. The highest frequency 6500 in the test is more than the fundamental and second resonant 
frequency simulated with COMSOL. Therefore, it can be drawn the designed device can function at stress modulated fundamental resonant frequency.

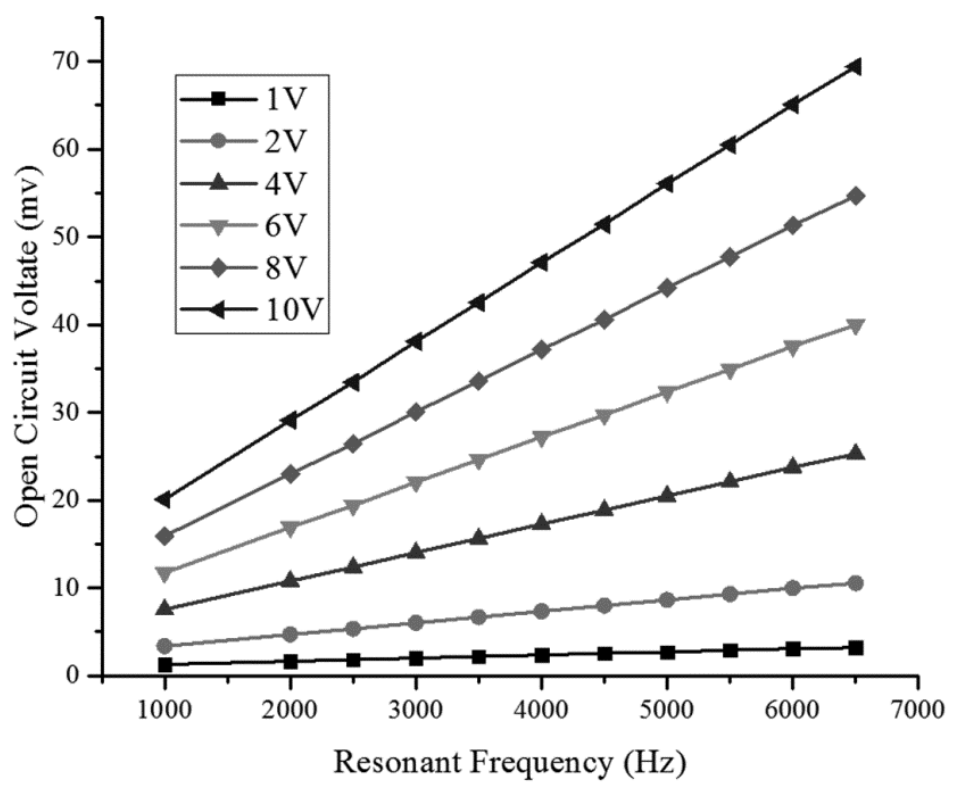

Fig. 9 Experimental results in acoustic-electric measurement system

Secondly, the device is tested in the wind tunnel. During the experiments it is necessary to convert alternative voltage into direct voltage. Thus, Wheatstone bridge circuit is used to convert voltage; capacitor is applied to store the energy produced by the films array; and Kiteley meter is applied for voltage monitoring which is shown in Fig. 4. The characteristics between wind velocity and open circuit voltage, which are shown in Fig. 10, exhibit that the maximum open circuit output voltage of the device is about $2.7 \mathrm{~V}$ when the wind velocity is more than $1 \mathrm{~m} / \mathrm{s}$. It is also illustrated that output voltage rises slowly along with the increase of wind velocity while decreases when the velocity more than $9.1 \mathrm{~m} / \mathrm{s}$.

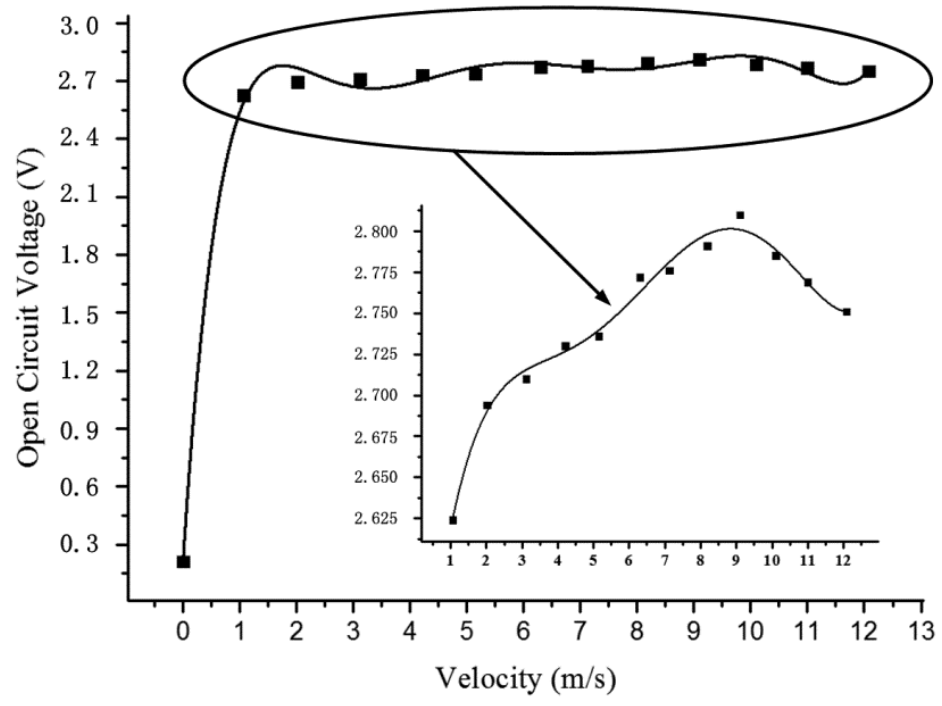

Fig. 10 The characteristic of wind velocity and open circuit voltage

The most important factor, which can be followed to analyze the problem of voltage decreasing, is the resonant frequency. It is shown in table 2 when the velocity is $9 \mathrm{~m} / \mathrm{s}$ the fundamental resonant frequency of thin 
film is about $11000 \mathrm{~Hz}$. It can be obtained by simulating the eigenfrequency of whole circular silicon device in COMSOL that the fundamental frequency of whole device is $11267 \mathrm{~Hz}$ which is shown in Fig.11. Compared two number it is found that the circular silicon plate begins to vibrate when the wind velocity is more than $9 \mathrm{~m} / \mathrm{s}$. At this moment the $\mathrm{ZnO}$ thin film lost their strong frame. When circular silicon plate deflected the surface of the circular silicon plate will increase. It means that the length of each frame of $\mathrm{ZnO}$ thin film will increase which will result in the decrease of the stress on $\mathrm{ZnO}$ thin film. According to equation (4), length increase and stress decrease will lead to decrease of fundamental resonant frequency. Therefore, the output voltage will reduce.

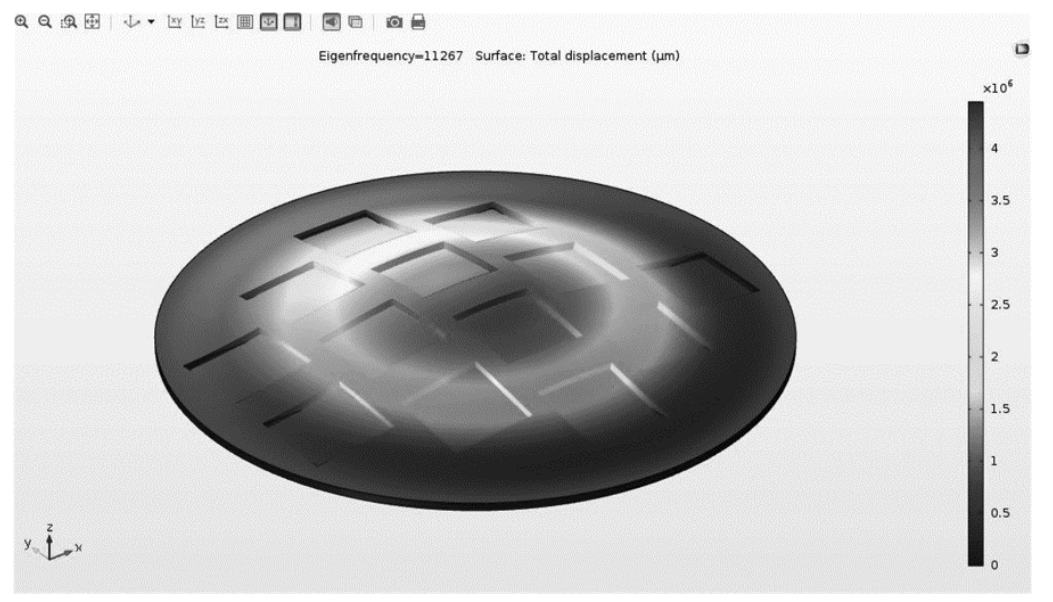

Fig. 11 The fundamental frequency of whole energy harvester

The performance of designed harvester is also illustrated by characteristics curve of load voltage of device under different wind velocity and characteristics curve of output power density of device with different load resist which are shown in Fig. 12 (a) and (b). It is observed that output voltage increases with the load resistance increasing. The trend is similar to the open circuit voltage under the fixed load resist. It is also revealed that more power will be obtain under higher load resistance. That is the device has good load capacity which the maximum output power density reaches $23.39 \mathrm{nW} / \mathrm{cm}^{2}$.

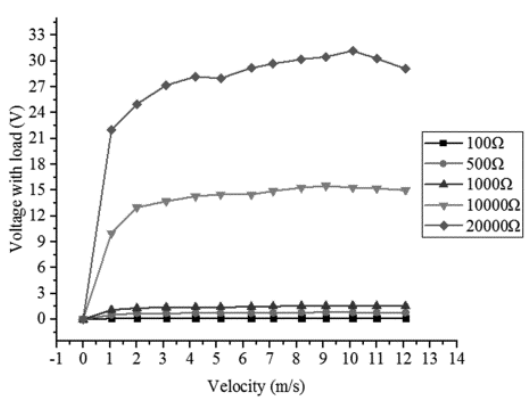

(a)

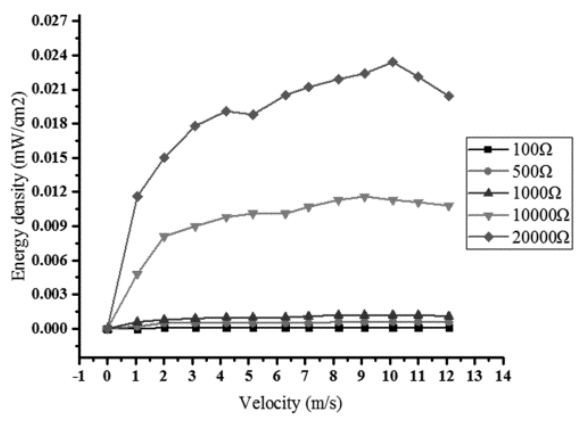

(b)

Fig. 12 Characteristics curve (a) load voltage of device in different wind velocity; (b) output power density of device with different load resist

In a conclusion, it is proved by the experimental results that the thin films can vibrate under a low wind velocity $1 \mathrm{~m} / \mathrm{s}$ and are easy to vibrate in wind turbulence. After the harvester beginning vibration the output voltage will change slowly with the variation of wind velocity.

In real scenario, wind flow is mainly existed as turbulent in nature because the Reynolds Number is more than 2300 due to the diameter is very large according to equation (6). The designed device is more easy to meet the vibration condition to generate energy. However, it cannot always generate power when the wind changes its 
direction. But it is true that there is definitely a building wall on which the wind directly blew. Hence, the designed device can function as the skin of building to generate energy. From the robustness point of view, the plate structure which is more robust than cantilever/beam structure, is more suitable to use as the building skin to generate energy. In a word, these characteristics of designed harvester enable it to be used as the building skin to harvest wind energy.

\section{Conclusion}

A wind energy harvester is developed with a circular silicon plate with several symmetrically distributed rectangular cavities which were covered with $\mathrm{ZnO}$ thin films based on MEMS technology. It can easily function when the wind velocity is more than $1 \mathrm{~m} / \mathrm{s}$. The maximum output power density of it reaches $23.39 \mathrm{nW} / \mathrm{cm}^{2}$. All of characteristics of designed harvester enable it to be used as the building skin to harvest wind energy. The relevant technology is promising for ambient wind energy harvesting on high buildings in city blocks. But there are more chances to enhance the performance of harvester. Firstly, material with higher piezoelectric coefficient (like PZT) can effectively increase the power density. Secondly, thicker silicon wafer as the frame of thin film can enhance the robust of harvester. All of them can extent the velocity ranges in which the output voltage will always increase.

\section{Acknowledgement}

We would like to express our sincere appreciation to the anonymous reviewers for their insightful comments, which have greatly improved the quality of the paper. Also, we would like to express our gratitude to anyone who helps us revise the paper.

This work is supported by National Natural Science Foundation of China (No.51305423) and China Scholarship Council.

\section{Reference}

[1] S.K. Salman and A.L.J. Teo, Windmill modeling consideration and factors influencing the stability of a grid-connected wind power-based embedded generator View Document, IEEE Transactions on Power Systems, 18(2) (2003) $793-802$.

[2] M. Chinchilla, S. Arnaltes and J.C. Burgos, Control of permanent-magnet generators applied to variable-speed wind-energy systems connected to the grid, IEEE Transactions on Energy Conversion, 21 (1) (2006) 130 - 135.

[3] Y. Bazilevs, M. C. Hsu and J. Kiendl, 3D simulation of wind turbine rotors at full scale. Part II: Fluid-structure interaction modeling with composite blades, Int J Numer Meth FL, 65 (2011) 236-253.

[4] B. Boukhezzar, and H. Siguerdidjane, Nonlinear control with wind estimation of a DFIG variable speed wind turbine for power capture optimization, Energy Conversion and Management, 50 (2009) 885-892.

[5] M. Huleihil, Maximum windmill efficiency in finite time, J. Appl. Phys., 105 (2009) 104908(1-4).

[6] D. A. Howey, A. Bansal, and A.S. Holmes, Design and performance of a centimeter-scale shrouded wind turbine for energy harvesting, Smart Material and Structure, 20 (2011) 1-12.

[7] H. J. Jung and S. W. Lee, the experimental validation of a new energy harvesting system based on the wake galloping phenomenon, Smart Material and Structure, 20 (2011) 055022(1-10).

[8] J.X. Zhao, J. Yang, Z.W. Lin, N. Zhao, J. Liu, Y.M. Wen, P. Li, An arc-shaped piezoelectric generator for multi-directional wind energy harvesting, Sensors and Actuators A, 236 (2015) 173-179

[9] J. Jun, F.R. Kong, L.G. He, Q.C. Guan and Z.H. Feng, Piezoelectric Wind-Energy-Harvesting Device with Reed and Resonant Cavity, Japan. Journal of Application Physics, Part 1, 49 (2010) 050204(1-3).

[10] S. Priya, C. T. Chen, and D. Fye, Piezoelectric windmill: a novel solution to remote sensing, Japan. J. Appl. Phys. 44 (2005) L104-L107.

[11] S.G. Li, J. P. Yuan and H. Lipson, Ambient wind energy harvesting using cross flow fluttering, Journal of Application Physics, 109 (2011) 026104(1-3).

[12] S.W. Ibrahim and W. G. Ali, a review on frequency tuning methods for piezoelectric energy harvesting 
systems, Journal Renewable Sustainable Energy, 4, (2012) 062703(1-29).

[13] H. C. Liu, C. J. Tay, C. G. Quan, T. Kobayashi, and C. K. Lee, Piezoelectric MEMS energy harvester for low-frequency vibrations with wideband operation range and steadily increased output power, J. Microelectromech. Syst, 20 (2011) 1131-1142.

[14] H. B. Fang, J. Q. Liu, and Z. Y. Xu, Fabrication and performance of MEMS-based piezoelectric power generator for vibration energy harvesting, Microelectronics Journal, 37(11) (2006) 1280-1284.

[15] D. N Shen, J. H Park, and J. Ajitsaria, the design, fabrication and evaluation of a MEMS PZT cantilever with an integrated Si proof mass for vibration energy harvesting, J. Micromech. Microeng, 18 (2008) 055017(1-7).

[16] S.H Li, A. Crovettoc, Z.T Penga and F Wang, etc., Bi-resonant structure with piezoelectric PVDF films for energy harvesting from random vibration sources at low frequency, Sensors and Actuators A, 247 (2016) 547-554.

[17] Z. L Wang, J. Song, Piezoelectric Nanogenerators Based on Zinc Oxide Nanowire Arrays, Science, 312 (2006) 242-246.

[18] X. Chen, S. Xu, N. Yao, Y. Shi, 1.6 V Nanogenerator for Mechanical Energy Harvesting Using PZT Nanofibers, Nano Lett., 10 (2010) 2133-2137.

[19] G.T Hwang, H. Park, J.H Lee, etc., Self-Powered Cardiac Pacemaker Enabled by Flexible Single Crystalline PMN-PT Piezoelectric Energy Harvester, Adv. Mater., 26 (2014) 4880-4887.

[20] K. Park, S. Xu, Y. Liu, G.-T. Hwang, S.-J. L. Kang, Z. L. Wang, K. J. Lee, Piezoelectric BaTiO3 Thin Film Nanogenerator on Plastic Substrates, Nano Lett., 10 (2010) 4939-4943.

[21] M. M Alam, S. K Ghosh, A. Sultana, D. Mandal, Lead-free ZnSnO3/MWCNTs-based self-poled flexible hybrid nanogenerator for piezoelectric power generation, Nanotechnology, 26 (2015) 165403.

[22] C. K Jeong, K. Park, J. Ryu, G. T Hwang, K. J Lee, Large-Area and Flexible Lead-Free Nanocomposite Generator Using Alkaline Niobate Particles and Metal Nanorod Filler, Adv. Funct. Mater., 24 (2014) 2620-2629.

[23] S. K Ghosh, T. K Sinha, B. Mahanty, and D. Mandal, Self-poled Efficient Flexible "Ferroelectretic" Nanogenerator: A New Class of Piezoelectric Energy Harvester, Energy Technol., 3 (2015) 1190 - 1197.

[24] S. K Ghosh, T.K Sinha, B. Mahanty, S. Jana, and D. Mandal, Porous polymer composite membrane based nanogenerator: A realization of self-powered wireless green energy source for smart electronics applications, Journal Of Applied Physics, 120 (2016) 174501.

[25] S. Jana, S. Garain, S. K. Ghosh, S. Sen and D. Mandal, The preparation of -crystalline non-electrically poled photoluminescant ZnO-PVDF nanocomposite film for wearable nanogenerators, Nanotechnology, 27 (2016) 445403.

[26] P. Adhikary, S. Garain, S. Ram, D. Mandal, Flexible Hybrid Eu31 Doped P(VDF-HFP) Nanocomposite Film Possess Hypersensitive Electronic Transitions and Piezoelectric Throughput, Journal Of Polymer Science, Part B: Polymer Physics, 54 (2016) 2335-2345.

[27] S. K Ghosh, A Biswas, S. Sen, C Das, K Henkel, D Schmeisser, D Mandal, Yb3+ assisted self-polarized PVDF based ferroelectretic nanogenerator: A facile strategy of highly efficient mechanical energy harvester fabrication, Nano Energy 30 (2016) 621-629.

[28] M. K Gupta, S.W Kim, and B. Kumar, Flexible High-Performance Lead-Free $\mathrm{Na}_{0.47} \mathrm{~K}_{0.47} \mathrm{Li}_{0.06} \mathrm{NbO}_{3}$ Microcube-Structure-Based Piezoelectric Energy Harvester, ACS Appl. Mater. Interfaces, 8 (2016) 1766-1773.

[29] S. K Ghosh, D. Mandal, Efficient natural piezoelectric nanogenerator: Electricity generation from fish swim bladder n, Nano Energy, 28(2016)356-365.

[30] S. H Wu, L. D Du, Z. Zhao, D. Y. Kong, Z. Fang, Novel Wind Energy Harvesting Device for City Building Based on ZnO Films Array, Key Engineering Materials 645-646 (2015) 1139-1144.

[31] R.D. Blevins, flow-induced vibration, Van Nostrand Reinhold Company, 1977.

[32] Y. Tamura, AIJ Recommendations for Loads on Buildings, Architectural Institute of Japan, 1996 Chapter 6, 1- 
80.

[33] H. Bau, N. F. DeRooij and B. K. Wolfgang, etc., Sensors, Mechanical Sensors (John Wiley \& Sons, 2008) Chapter 5, 166.

[34] R. Portugal, L. Golebiowski and D. Frenkel, Oscillation of membranes using computer algebra, Am. J. Phys. 67 (6) (1999) 534-537.

[35] S. H. Ho and C. K. Chen, Free vibration analysis of non-homogeneus rectangular membranes using a hybrid method, J Sound Vib, 233 (3) (2000) 547-555.

[36] W. K. Schomburg, Introduction to Microsystem Design second edition, Springer-Verlag Beelin Heidelberg, 2015 Chapter vibration, pp.111.

[37] B. Liu, X. Feng, S.M. Zhang, The effective Young's modulus of composites beyond the Voigt estimation due to the Poisson effect, Compos Sci Technol. 69 (2009) 2198-2204.

[38] S. Barta, Effective Young's modulus and Poisson's ratio for the particulate composite, J. Appl. Phys. 75 (1994) 3258-3263. 
Biographies

Lidong Du was born in 1980. He received his Ph.D. at the Institute of Electronics, Chinese Academy of Sciences. Since 2010, he has worked in the State Key Laboratory of Transducer Technology (SKLTT) at Institute of Electronics, Chinese Academy of Sciences. Now he is an Academic visitor in Nano Group, Electronics and Computer Science, University of Southampton, UK from April 2016. His major field is integration technology and system of micro sensors.

Zhen Fang was born in 1976. He received his $\mathrm{PhD}$ at the Institute of Electronics, Chinese Academy of Sciences. He has been a professor in the State Key Laboratory of Transducer Technology at the Institute of Electronics, Chinese Academy of Sciences. His major field is wireless sensor networks and microsystems of integrate sensors.

Jize Yan was born in 1982. He received the first degree from Tsinghua University in China, and Ph.D. degree from the University of Cambridge. Since 2007, he was a Research Assistant, Research Associate, Senior Research Associate and Principal Investigator in the University of Cambridge. Now he is an Associate Professor in the University of Southampton. His research work led to 80+ peer-reviewed publications; served as an editorial board member for several international journals, and a reviewer of 18 journals. His research field related to Wireless Sensor Network, Energy Harvesting, distributed optical sensor network and silicon photonics.

Zhan Zhao was born in 1958. He received his B.S. in Physics in 1982 from Shanxi University and his master's and doctoral degrees in Physical Electronics and Devices in 1987 and 2003, respectively. Both the degrees were awarded by the Institute of Electronics, Chinese Academy of Sciences (IECAS). Since 1994, he has been with the State Key Laboratory of Transducer Technology (SKLTT) at IECAS. He was a Visiting Scholar in Rutherford Appleton Laboratory, UK, in 2001. Since 2000, he has been a professor for integrated sensor and microsystems at the SKLTT. 